\title{
Travel-acquired infections in Canada: CanTravNet 2011-2012
}

\author{
Boggild $A K^{1,2^{*}}$, Geduld $\mathrm{J}^{3}$, Libman $\mathrm{M}^{4}$, Ward $\mathrm{BJ}^{4}$, McCarthy $\mathrm{A}^{5}$, Hajek $\mathrm{J}^{6}$, Ghesquiere $\mathbf{W}^{7}$, Vincelette $\mathrm{J}^{8}$, \\ Kuhn $S^{9}$, Freedman $D O^{10}$ and Kain $\mathrm{KC}^{1,11}$
}

\footnotetext{
${ }^{1}$ Tropical Disease Unit, Department of Medicine, University Health Network and the University of Toronto, Toronto, ON

${ }^{2}$ Public Health Ontario Laboratories, Public Health Ontario, Toronto, ON

${ }^{3}$ Travel and Migration Health Division, Public Health Agency of Canada, Ottawa, ON

${ }^{4}$ Division of Infectious Diseases, McGill University Health Centre, Montreal, QC

${ }^{5}$ Tropical Medicine and International Health Clinic, Ottawa Hospital and the University of Ottawa, Ottawa, ON

${ }^{6}$ Division of Infectious Diseases, Vancouver General Hospital and University of British Columbia, Vancouver, BC

${ }^{7}$ Infectious Diseases, Vancouver Island Health Authority and University of British Columbia, Victoria, BC

${ }^{8}$ Hôpital Saint-Luc du CHUM and Université de Montréal, Montréal, QC

${ }^{9}$ Division of Pediatric Infectious Diseases, Alberta Children's Hospital and the University of Calgary, Calgary, AB

${ }^{10}$ Gorgas Center for Geographic Medicine, University of Alabama Birmingham, Birmingham, AL

${ }^{11}$ SAR Laboratories, Sandra Rotman Centre for Global Health, Toronto, ON

*Corresponding author: andrea.boggild@utoronto.ca
}

\section{Abstract}

Background: Important gaps remain in our knowledge of the infectious diseases people acquire while travelling and the impact of pathogens imported by Canadian travellers.

Objective: To provide a surveillance update of illness in a cohort of returned Canadian travellers and new immigrants.

Methods: Data on returning Canadian travellers and new immigrants presenting to a CanTravNet site between September 2011 and September 2012 were extracted and analyzed by destination, presenting symptoms, common and emerging infectious diseases and disease severity.

Results: During the study period, 2283 travellers and immigrants presented to a CanTravNet site, $88 \%(\mathrm{~N}=2004)$ of whom were assigned a travel-related diagnosis. Top three destinations for non-immigrant travellers were India $(\mathrm{N}=132)$, Mexico $(\mathrm{N}=103)$ and Cuba $(\mathrm{N}=89)$. Fifty-one cases of malaria were imported by ill returned travellers during the study period, $60 \%(\mathrm{~N}=30)$ of which were Plasmodium falciparum infections. Individuals travelling to visit friends and relatives accounted for $83 \%$ of enteric fever cases (15/18) and $41 \%$ of malaria cases $(21 / 51)$. The requirement for inpatient management was over-represented among those with malaria compared to those without malaria $(25 \%$ versus $2.8 \% ; p<0.0001)$ and those travelling to visit friends and relatives versus those travelling for other reasons $(12.1 \%$ versus $2.4 \%$; $p<0.0001)$. Nine new cases of HIV were diagnosed among the cohort, as well as one case of acute hepatitis $B$. Emerging infections among travellers included hepatitis $E$ virus $(\mathrm{N}=6)$, chikungunya fever $(\mathrm{N}=4)$ and cutaneous leishmaniasis $(\mathrm{N}=16)$. Common chief complaints included gastrointestinal $(\mathrm{N}=804)$, dermatologic $(\mathrm{N}=440)$ and fever $(\mathrm{N}=287)$. Common specific causes of chief complaint of fever in the cohort were malaria ( $N=47 / 51$ total cases), dengue fever (14/18 total cases), enteric fever ( $14 / 17$ total cases) and influenza and influenza-like illness (15/21 total cases). Animal bites were the tenth most common diagnosis among tourist travellers. 
Interpretation: Our analysis of surveillance data on ill returned Canadian travellers provides a recent update to the spectrum of imported illness among travelling Canadians. Preventable travel-acquired illnesses and injuries in the cohort include malaria, enteric fever, HIV, hepatitis B, hepatitis A, influenza and animal bites. Strategies to improve uptake of preventive interventions such as malaria chemoprophylaxis, immunizations and arthropod/animal avoidance may be warranted.

\section{Introduction}

In 2012, Canadians spent $\$ 36.5$ billion on international tourism, up from $\$ 35.9$ billion the year before (1). Top tourist destinations for Canadians continue to include tropical and economically developing countries such as Mexico, Cuba, the Dominican Republic and Jamaica (2). In 2012, of the 11,363,100 Canadians who stayed one or more nights at a destination other than the United States, almost a third travelled to one of those four countries.

International tropical travel puts travellers at risk of enteric and vector-borne infectious diseases (3-5), many of which are preventable through specific interventions such as chemoprophylaxis, immunization, insect repellents, personal protective measures and avoidance (4). A large-scale analysis of illness in returned Canadian travellers and new immigrants over a two-year period has recently been published by members of CanTravNet (6), provides Canadian practitioners with an epidemiologic roadmap of travel-acquired infections, which can be used to inform decision-making in both the pre-travel and post-travel setting.

This surveillance report provides an update to the two-years' worth of CanTravNet data published previously (6) and highlights the breadth of illnesses encountered by Canadians visiting $>130$ countries over a one-year period and presenting for care at a CanTravNet site.

\section{Methods}

\section{Data sources}

Six Canadian sites from four provinces (British Columbia, Alberta, Ontario and Quebec) also belonging to the GeoSentinel Global Surveillance Network have grouped together to form the core sites of CanTravNet (6). The six sites in Canada are large referral-based outpatient centres that primarily service the Greater Vancouver/Victoria, Calgary, Toronto, Ottawa and Montreal areas, which account for $47 \%$ of the Canadian population (or, a catchment of $\sim 15.5$ million people). They are staffed by specialists in travel and tropical medicine and immediate referral from the affiliated emergency departments is common.

Data were collected using the GeoSentinel data platform. This network is comprised of 56 specialized travel/tropical medicine clinics on six continents, which contribute denominalized clinician- and questionnairebased travel surveillance data on all ill travellers examined to a centralized Structured Query Language database (7). (For additional details see http://www.istm.org/geosentinel ). Collected data include patient demographics, details of recent travel, five-year travel history, purpose of travel and presence of absence of a pre-travel encounter with a healthcare provider. Final diagnoses are made by attending physicians and assigned a diagnostic code selected from a standardized list of $>500$ diagnostic entities, including etiologic (e.g. Giardia) and syndromic (e.g. cough) diagnoses. Syndromic codes are entered where an etiologic code cannot be assigned due to use of empiric therapy, self-limited disease, or inability to justify a more extensive workup as part of routine clinical practice. All CanTravNet sites contribute microbiologically confirmed data, where available, based on the best national reference diagnostic tests (including molecular diagnostics) available at the time. 'Probable' diagnoses are restricted to patients with pathognomonic physical findings (e.g. tick eschar), clinical response to highly specific therapy, or classical presentation and exposure history with laboratory exclusion of other possible etiologies (6). Further details regarding CanTravNet can be found at http://www.istm.org/cantravnet. 


\section{Definitions and classifications}

Reason for most recent travel. Six possible travel purpose designations are available: immigration (including refugee), tourism, business, missionary/volunteer research/aid work, visiting friends and relatives, and "other" (students, military personnel and medical tourists). A visiting friends and relatives (VFR) traveller is defined as an immigrant who is ethnically and/or racially distinct from the majority population in their current country of residence and who returns to his homeland to "visit friends and relatives". VFR travellers also include children of foreign-born parents (e.g. second generation immigrants) who return to their parent's homeland to visit friends and relatives. A VFR traveller designation is typically applied to individuals travelling from a high-income country of current residence to a low-income country of origin (8). "Medical tourists" are defined as those for whom the primary purpose of travel is to seek health care, and as a consequence of travel, acquire an infectious complication secondary to the medical care received or become ill with an infectious or non-infectious disease while abroad.

Countries of exposure and travel were assigned 14 regional classifications: North America, Central America, the Caribbean, South America, Western Europe, Eastern Europe, the Middle East, North Africa, Sub-Saharan Africa, South Central Asia, Southeast Asia, Northeast Asia, Australia/New Zealand and Oceania.

\section{Inclusion criteria}

Demographic, clinical and travel-related data on Canadian citizens and new immigrants to Canada encountered after completion of their international travel or residence abroad and seen in any of five CanTravNet sites from September 2011 to September 2012 were extracted and analyzed. (The Calgary site was new to GeoSentinel as of 2012 and did not contribute cases during the study period.) Only patients with probable or confirmed final diagnoses (specific etiology or syndrome as described previously (6)) were included.

\section{Descriptive analysis}

Extracted data were managed in a Microsoft Access database and analyzed using standard parametric and nonparametric techniques. Travellers were described by purpose of travel, demographics, diagnoses, country of exposure and region of travel. Top syndromic and etiologic diagnoses were described for each purpose of travel. Top chief complaints were described by represented causative diagnoses and top source countries. Comparisons between categorical variables (e.g. purpose of travel) were made using Yates' corrected chi-square analysis, while continuous variables (e.g. age) were analyzed for significant differences using the Student's t-test and in the case of non-normally distributed parameters, the Mann-Whitney Rank Sum test. For a particular variable (e.g. purpose of travel or diagnosis), the reference population was all other travellers in the cohort without that variable (e.g. malaria versus non-malaria). Differences between groups of continuous variables were compared using One Way ANOVA or Kruskal Wallis One Way ANOVA on ranks. All statistical computations were performed using SigmaStat 2.03 software (SPSS Inc., Chicago, IL). Level of significance was set at $p<0.05$.

\section{Results}

\section{Patients and demographics}

For the surveillance period reported, the cohort of 2283 travellers who presented to a CanTravNet site was assigned 2377 confirmed and 338 probable diagnoses. Of the 2283 travellers seen, $2004(87.8 \%)$ had a travelrelated diagnosis (hereafter referred to as "ill returned travellers"), $166(7.3 \%)$ had a non-travel-related diagnosis and $113(4.9 \%)$ had a diagnosis whose relatedness to travel could not be ascertained. The cohort of 2283 travellers presented to one of five CanTravNet sites as follows: Montreal-McGill ( $\mathrm{N}=955,41.8 \%)$, Toronto $(\mathrm{N}=521$, 22.8\%), Ottawa ( $\mathrm{N}=451,19.8 \%)$, Montreal-Centre Hospitalier de l'Université de Montreal (CHUM) ( $N=245,10.7 \%)$ 
and Vancouver/Victoria ( $\mathrm{N}=111,4.9 \%)$. Major demographic variables for the cohort of 2004 travellers with travelrelated diagnoses are summarized in Table 1. Top countries of birth for individuals born outside of Canada $(\mathrm{N}=915)$ were: India $(\mathrm{N}=82,9 \%)$, China $(\mathrm{N}=42,4.6 \%)$, Philippines $(\mathrm{N}=41,4.5 \%)$, France $(\mathrm{N}=38,4.2 \%)$ and the United States ( $\mathrm{N}=32,3.5 \%)$, with 129 represented countries.

Non-immigration travellers in the cohort (i.e. all travellers in the cohort except those travelling for the purpose of immigration) $(\mathrm{N}=1511 / 2004)$ for whom exposure country was known $(\mathrm{N}=1349)$ visited 133 different countries, the most frequently visited of which included: India $(N=132,9.8 \%)$, Mexico $(N=103,7.6 \%)$, Cuba $(N=89,6.6 \%)$, Dominican Republic $(\mathrm{N}=71,5.3 \%)$ and Thailand $(\mathrm{N}=49,3.6 \%)$.

Table 1: Demographic and clinical characteristics of 2004 returned travellers or new immigrants presenting to a CanTravNet site for care of a travel-related illness, 2011-2012*

\begin{tabular}{|c|c|c|c|c|c|c|c|c|c|c|c|c|c|c|}
\hline \multirow[b]{2}{*}{ Characteristic } & \multirow{2}{*}{\multicolumn{2}{|c|}{$\begin{array}{l}\text { All } \\
\text { travellers } \\
n=2004\end{array}$}} & \multicolumn{10}{|c|}{ Purpose of travel; no. (\%) of travellers ${ }^{\ddagger}$} & & \\
\hline & & & \multicolumn{2}{|c|}{$\begin{array}{c}\text { Tourism } \\
n=883\end{array}$} & \multicolumn{2}{|c|}{$\begin{array}{c}\text { Immigration } \\
n=493\end{array}$} & \multicolumn{2}{|c|}{$\begin{array}{c}\text { Visit friends } \\
\text { and relatives } \\
n=206\end{array}$} & \multicolumn{2}{|c|}{$\begin{array}{c}\text { Missionary, } \\
\text { volunteer, } \\
\text { researcher, } \\
\text { aid worker } \\
n=212\end{array}$} & \multicolumn{2}{|c|}{$\begin{array}{c}\text { Business } \\
n=160\end{array}$} & \multicolumn{2}{|c|}{$\begin{array}{l}\text { Other }^{\S} \\
n=50\end{array}$} \\
\hline Male & 856 & 42.7 & 327 & 37.0 & 240 & 48.7 & 101 & 49.0 & 73 & 34.4 & 97 & 60.6 & 18 & 36.0 \\
\hline Female & 1147 & 57.2 & 556 & 63.0 & 252 & 51.1 & 105 & 51.0 & 139 & 65.6 & 63 & 39.4 & 32 & 64.0 \\
\hline Unknown & 1 & & & & 1 & 0.2 & & & & & & & & \\
\hline Age, yr, median (range) & 38 & $0-87$ & 38 & $1-81$ & 39 & $1-84$ & 40 & $0-87$ & 30 & $15-79$ & 41 & $22-78$ & 25 & $9-70$ \\
\hline \multicolumn{15}{|l|}{ Type of patient } \\
\hline Inpatient & 68 & 3.4 & 13 & 1.5 & 20 & 4.1 & 25 & 12.1 & 4 & 1.9 & 5 & 3.1 & 1 & 2.0 \\
\hline Outpatient & 1936 & 96.6 & 870 & 98.5 & 473 & & 181 & & 208 & & 155 & & 49 & \\
\hline $\begin{array}{l}\text { Travel duration, } d, \\
\text { median (range) }\end{array}$ & 20 & $0-3660$ & 15 & $0-3660$ & NA & NA & 36 & $1-553$ & 37 & $3-3659$ & 21.5 & $1-1339$ & 46 & $3-2526$ \\
\hline \multicolumn{15}{|l|}{$\begin{array}{l}\text { Pre-travel medical } \\
\text { encounter }\end{array}$} \\
\hline Yes & 594 & 29.6 & 299 & 33.9 & NA & NA & 33 & 16.0 & 143 & 67.5 & 83 & 51.9 & 29 & 58.0 \\
\hline No & 656 & 32.7 & 362 & 41.0 & NA & NA & 116 & 56.3 & 24 & 11.3 & 40 & 25.0 & 14 & 28.0 \\
\hline Unknown & 754 & 37.6 & 222 & 25.1 & NA & NA & 57 & 27.7 & 45 & 21.2 & 37 & 23.1 & 7 & 14.0 \\
\hline \multicolumn{15}{|l|}{ Syndromic diagnoses } \\
\hline Gastrointestinal & 986 & 49.2 & 456 & 51.6 & 200 & 40.6 & 91 & 44.2 & 114 & 53.8 & 93 & 58.1 & 32 & 64.0 \\
\hline Dermatologic & 434 & 21.7 & 311 & 35.2 & 15 & 3.0 & 35 & 17.0 & 37 & 16.0 & 25 & 15.6 & 11 & 22.0 \\
\hline Systemic febrile illness & 225 & 11.2 & 70 & 7.9 & 36 & 7.3 & 60 & 29.1 & 28 & 13.2 & 28 & 17.5 & 3 & 6.0 \\
\hline Respiratory & 113 & 5.6 & 41 & 4.6 & 35 & 7.1 & 15 & 7.3 & 9 & 4.2 & 12 & 7.5 & 1 & 2.0 \\
\hline \multicolumn{15}{|l|}{$\begin{array}{l}\text { Geographic region of } \\
\text { exposure }\end{array}$} \\
\hline Sub-Saharan Africa & 444 & 22.2 & 76 & 8.6 & 141 & 28.6 & 58 & 28.2 & 105 & 49.5 & 52 & 32.5 & 22 & 44.0 \\
\hline Caribbean & 304 & 15.2 & 245 & 27.7 & 32 & 6.5 & 14 & 6.8 & 25 & 11.8 & 13 & 8.1 & 1 & 2.0 \\
\hline South Central Asia & 278 & 13.9 & 73 & 8.3 & 104 & 21.1 & 71 & 34.5 & 13 & 6.1 & 11 & 6.9 & 9 & 18.0 \\
\hline Central America & 214 & 10.7 & 182 & 20.6 & 4 & 0.8 & 11 & 5.3 & 15 & 7.1 & 16 & 10.0 & 7 & 14.0 \\
\hline South East Asia & 206 & 10.3 & 112 & 12.7 & 69 & 14.0 & 10 & 4.9 & 9 & 4.2 & 11 & 6.9 & 0 & 0 \\
\hline South America & 123 & 6.1 & 58 & 6.6 & 14 & 2.8 & 20 & 9.7 & 14 & 6.6 & 13 & 8.1 & 6 & 12.0 \\
\hline North East Asia & 74 & 3.7 & 16 & 1.8 & 44 & 8.9 & 7 & 3.4 & 1 & 0.5 & 8 & 5.0 & 1 & 2.0 \\
\hline North America & 51 & 2.5 & 42 & 4.8 & 1 & 0. & 0 & 0 & 1 & 0.5 & 6 & 3.8 & 1 & 2.0 \\
\hline
\end{tabular}




\begin{tabular}{l|c|c|c|c|c|c|c|c|c|c|c|c|c|c} 
Western Europe & 34 & 1.7 & 24 & 2.7 & 7 & 1.4 & 1 & 0.5 & 1 & 0.5 & 1 & 0.6 & 0 & 0 \\
\hline Eastern Europe & 38 & 1.9 & 3 & 0.3 & 27 & 5.5 & 6 & 2.9 & 1 & 0.5 & 0 & 0 & 1 & 2.0 \\
\hline Middle East & 36 & 1.8 & 8 & 0.9 & 21 & 4.3 & 2 & 1.0 & 0 & 0 & 5 & 3.1 & 0 & 0 \\
\hline North Africa & 41 & 2.0 & 10 & 1.1 & 19 & 3.9 & 4 & 1.9 & 4 & 1.9 & 6 & 3.8 & 0 & 0 \\
\hline Australia / New Zealand & 5 & 0.2 & 3 & 0.3 & 0 & 0 & 0 & 0 & 0 & 0 & 1 & 0.6 & 1 & 2.0 \\
\hline Oceania & 8 & 0.4 & 4 & 0.5 & 2 & 0.4 & 0 & 0 & 2 & 0.9 & 0 & 0 & 0 & 0 \\
\hline Unknown & 148 & 7.4 & 99 & 11.2 & 8 & 1.6 & 2 & 1.0 & 21 & 9.9 & 17 & 10.6 & 1 & 2.0 \\
\hline Birth country & & & & & & & & & & & & & & \\
\hline Canada & 1089 & 54.3 & 732 & 82.9 & 0 & 0 & 42 & 20.4 & 161 & 75.9 & 117 & 73.1 & 36 & 72.0 \\
\hline Outside Canada & 915 & 45.7 & 151 & 17.1 & 493 & 100.0 & 164 & $79.6^{\dagger}$ & 51 & 24.1 & 43 & 26.9 & 14 & 28.0
\end{tabular}

${ }^{*}$ The cohort consisted of 2004 travellers with a definitive travel-related diagnosis, 166 with a non-travel-related diagnosis and 113 with a diagnosis for which relation to travel could not be ascertained. This analysis includes only those travellers with a travel-related diagnosis.

${ }^{\ddagger}$ Except where indicated otherwise.

SIncludes students $(n=42)$, military personnel $(n=6)$ and medical tourists $(n=2)$.

${ }^{\dagger}$ Among those born outside of Canada, people who travelled for the purpose of visiting friends and relatives were defined as immigrants who were ethnically and/or racially distinct from the majority population in their current country of residence and who returned to their homeland to visit friends and relatives. This group also included children of foreign-born parents (e.g. second generation immigrants) who returned to their parents' homeland to visit friends and relatives.

\section{Diagnoses}

A total of 2402 travel-related diagnoses were issued to 2004 ill returned travellers. Of these diagnoses, 2078 were confirmed and 324 were probable. The most frequently issued travel-related diagnoses in persons travelling for the purpose of immigration were: latent tuberculosis, chronic hepatitis $B$, active tuberculosis, chronic hepatitis $C$ and strongyloidiasis (Table 2). The most frequently issued travel-related diagnoses among non-immigration travellers were: post-infectious irritable bowel syndrome, acute diarrhea, chronic diarrhea, skin and soft tissue infections and arthropod bites (Table 2). Table 3 lists the top travel-related diagnoses and countries of exposure for travellers presenting with a chief complaint of fever, gastrointestinal symptoms and skin disease. Table 4 describes cases of malaria among the 2004 ill returned travellers.

\section{Table 2: Top 10 syndromic and etiologic diagnoses by reason for travel among 2004 ill returned travellers with definitive travel-related diagnoses, 2011-2012}

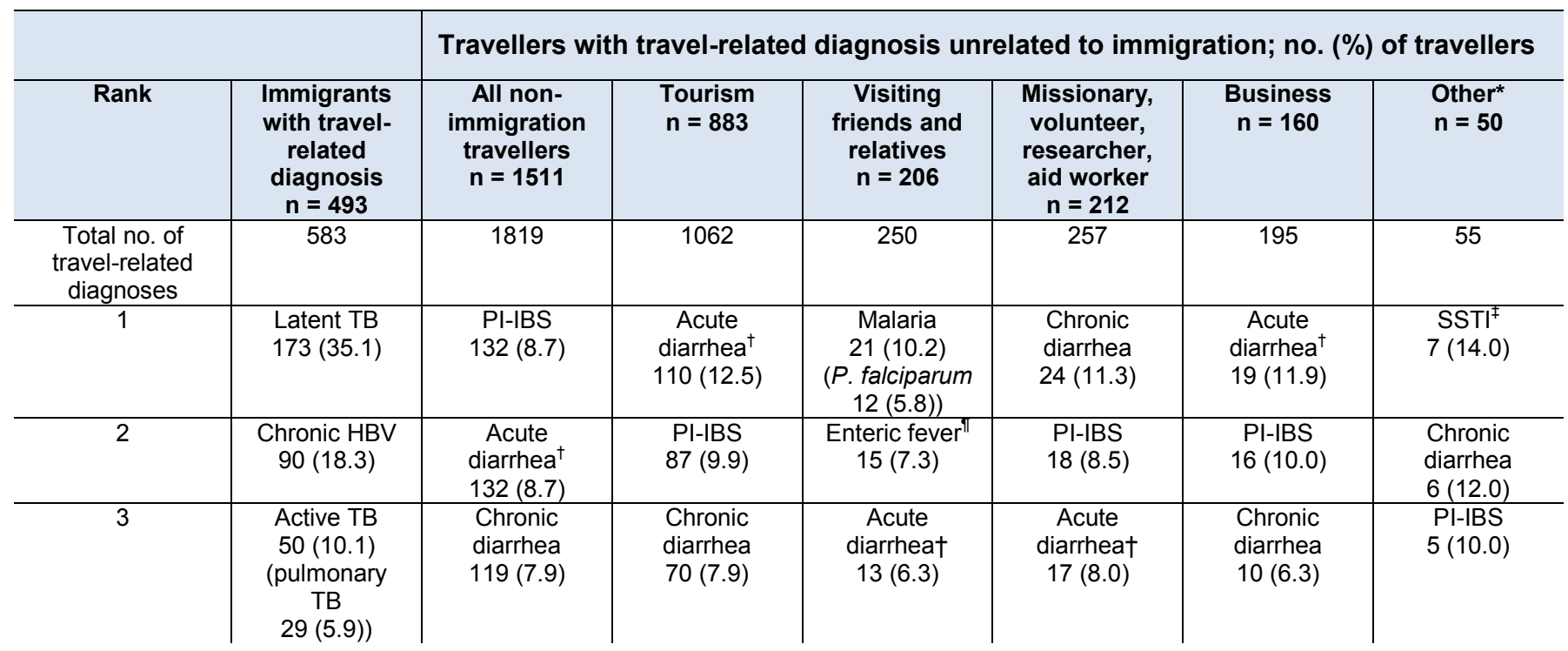




\begin{tabular}{|c|c|c|c|c|c|c|c|}
\hline 4 & $\begin{array}{c}\text { Chronic HCV } \\
33(6.7)\end{array}$ & $\begin{array}{c}\mathrm{SSTI}^{\ddagger} \\
83(5.5)\end{array}$ & $\begin{array}{c}\text { Arthropod bite } \\
65(7.4)\end{array}$ & $\begin{array}{c}\text { Chronic } \\
\text { diarrhea } \\
9(4.4) \\
\end{array}$ & $\begin{array}{c}S S T I^{\ddagger} \\
13(6.1)\end{array}$ & $\begin{array}{c}\text { Viral } \\
\text { syndrome } \\
10(6.3) \\
\end{array}$ & $\begin{array}{c}\text { Acute } \\
\text { diarrhea }^{\dagger} \\
5(10.0)\end{array}$ \\
\hline 5 & $\begin{array}{c}\text { Strongyloidiasis } \\
25(5.1)\end{array}$ & $\begin{array}{c}\text { Arthropod bite } \\
79(5.2)\end{array}$ & $\begin{array}{c}\text { Rash } \\
52(5.9)\end{array}$ & $\begin{array}{c}\text { Febrile illness } \\
<3 \text { wk } \\
\text { duration } \\
7(3.4)\end{array}$ & $\begin{array}{c}\text { Malaria } \\
12(5.7) \\
(P . \text { falciparum } \\
9(4.2))\end{array}$ & $\begin{array}{l}\text { Malaria } \\
9(5.6) \\
(P . \text { falciparum } \\
5(3.1))\end{array}$ & $\begin{array}{c}\text { Giardiasis } \\
4(8.0)\end{array}$ \\
\hline 6 & $\begin{array}{l}\text { Filariasis } \\
22(4.5)\end{array}$ & $\begin{array}{c}\text { Rash } \\
74(4.9)\end{array}$ & $\begin{array}{c}\text { SSTI }^{\ddagger} \\
50(5.7)\end{array}$ & $\begin{array}{c}\text { Rash } \\
7(3.4)\end{array}$ & $\begin{array}{c}\text { Abdominal } \\
\text { pain / } \\
\text { dyspepsia } \\
12(5.7) \\
\end{array}$ & $\begin{array}{c}\text { Rash } \\
7(4.4)\end{array}$ & $\begin{array}{c}\text { Latent TB } \\
3(6.0)\end{array}$ \\
\hline 7 & $\begin{array}{l}\text { Hydatid } \\
17(3.4)\end{array}$ & $\begin{array}{c}\text { Abdominal } \\
\text { pain / } \\
\text { dyspepsia } \\
62(4.1)\end{array}$ & $\begin{array}{l}\text { Cutaneous } \\
\text { larva migrans } \\
40(4.5)\end{array}$ & $\begin{array}{l}\text { PI-IBS } \\
6(2.9)\end{array}$ & $\begin{array}{c}\text { Latent TB } \\
11(5.2)\end{array}$ & $\begin{array}{c}\text { Blastocystis } \\
7(4.4)\end{array}$ & $\begin{array}{c}\text { Abdominal } \\
\text { pain / } \\
\text { dyspepsia } \\
3(6.0) \\
\end{array}$ \\
\hline 8 & $\begin{array}{c}\text { Schistosomiasis } \\
17(3.4)\end{array}$ & $\begin{array}{c}\text { Giardiasis } \\
47(3.1)\end{array}$ & $\begin{array}{c}\text { Abdominal } \\
\text { pain / } \\
\text { dyspepsia } \\
39(4.4)\end{array}$ & $\begin{array}{l}\text { SSTI }^{\ddagger} \\
5(2.4)\end{array}$ & $\begin{array}{c}\text { Febrile illness } \\
<3 \text { wk } \\
\text { duration } \\
8(3.8)\end{array}$ & $\begin{array}{c}\text { Giardiasis } \\
6(3.8)\end{array}$ & $\begin{array}{c}\text { Blastocystis } \\
2(4.0)\end{array}$ \\
\hline 10 & $\begin{array}{l}\text { Leprosy } \\
10(2.0) \\
\end{array}$ & $\begin{array}{c}\text { Blastocystis } \\
45(3.0)\end{array}$ & $\begin{array}{c}\text { Animal bite } \\
23(2.6)\end{array}$ & $\begin{array}{c}\text { Blastocystis } \\
5(2.4) \\
\end{array}$ & $\begin{array}{c}\text { Giardiasis } \\
6(2.8) \\
\end{array}$ & $\begin{array}{c}\text { Dientamoebiasis } \\
4(2.5)\end{array}$ & $\begin{array}{l}\text { Rash } \\
2(4.0)\end{array}$ \\
\hline
\end{tabular}

$\mathrm{HBV}=$ hepatitis $\mathrm{B}$ virus, $\mathrm{HCV}=$ hepatitis $\mathrm{C}$ virus, $\mathrm{P}$. falciparum = Plasmodium falciparum, PI-IBS = post-infectious irritable bowel syndrome, SSTI = skin and soft tissue infection, $\mathrm{TB}=$ tuberculosis, URTI = upper respiratory tract infection.

*Includes students $(n=42)$, military personnel $(n=6)$ and medical tourists $(n=2)$.

${ }^{\dagger}$ Includes acute bacterial, parasitic and viral diarrhea, as well as acute diarrhea of unspecified etiology.

${ }^{\ddagger}$ Includes erysipelas, cellulitis, furunculosis, carbuncles, skin abscess, pyoderma, ecthyma, impetigo and superficial fungal skin infections.

$\S$ Includes bites by cats, dogs, monkeys and other animals and monkey scratches.

"Includes infection with Salmonella enterica serotype Typhi or Paratyphi or Typhoid fever unspecified.

Table 3: Top diagnoses and source countries for specific etiologies within syndromic chief complaints among 2004 ill returned travellers with definitive travel-related diagnoses

\begin{tabular}{|c|c|c|c|c|}
\hline \multirow{2}{*}{$\begin{array}{l}\text { Diagnosis } \\
\text { Chief complaint fever }(n=287 \text { ) }\end{array}$} & \multicolumn{2}{|c|}{ No. (\%) of patients* } & \multirow{2}{*}{$\begin{array}{l}\text { Total no. } \\
\text { in } \\
\text { database } \\
\text { (travel- } \\
\text { related) }\end{array}$} & \multirow[t]{2}{*}{$\begin{array}{c}\text { Top three }{ }^{£} \text { source countries } \\
\text { for diagnosis }\end{array}$} \\
\hline & & & & \\
\hline Malaria & 47 & 92.2 & 51 & India, Nigeria, Sierra Leone \\
\hline Plasmodium falciparum & 28 & 93.3 & 30 & Nigeria, Haiti \\
\hline Severe cerebral & 1 & 100.0 & 1 & \\
\hline Plasmodium vivax & 11 & 91.7 & 12 & India, Pakistan, Afghanistan \\
\hline Plasmodium species unknown & 6 & 100.0 & 6 & \\
\hline Plasmodium ovale & 1 & 50.0 & 2 & Nigeria, South Sudan \\
\hline Dengue fever & 14 & 77.8 & 18 & Vietnam, Thailand, Guyana \\
\hline Active tuberculosis & 17 & 29.8 & 57 & $\begin{array}{l}\text { India, Philippines, China, } \\
\text { Vietnam }\end{array}$ \\
\hline Pulmonary & 7 & 20.6 & 34 & \\
\hline Extrapulmonary & 10 & 43.5 & 23 & \\
\hline
\end{tabular}




\begin{tabular}{|c|c|c|c|c|}
\hline Enteric fever & 14 & 82.4 & 17 & India, Pakistan \\
\hline $\begin{array}{l}\text { Salmonella enterica serotype } \\
\text { Typhi }\end{array}$ & 7 & 77.8 & 9 & \\
\hline $\begin{array}{l}\text { Salmonella enterica serotype } \\
\text { Paratyphi }\end{array}$ & 4 & 100.0 & 4 & \\
\hline Typhoid fever, unspecified & 3 & 75.0 & 4 & \\
\hline Influenza / Influenza-like illness & 15 & 71.4 & 21 & $\begin{array}{l}\text { Peru, India, Dominican } \\
\text { Republic }\end{array}$ \\
\hline Upper respiratory tract infection & 9 & 45.0 & 20 & India, Dominican Republic \\
\hline Pneumonia & 9 & 56.3 & 16 & China, Thailand \\
\hline Lobar & 7 & 53.8 & 13 & \\
\hline Atypical & 2 & 66.7 & 3 & \\
\hline Acute urinary tract infection & 5 & 50.0 & 10 & India \\
\hline Rickettsioses, spotted fever $^{\dagger}$ & 7 & 87.5 & 8 & $\begin{array}{l}\text { South Africa, India, Malawi, } \\
\text { Namibia }\end{array}$ \\
\hline Chikungunya fever & 2 & 50.0 & 4 & $\begin{array}{l}\text { India, Pakistan, Cambodia, } \\
\text { Kenya }\end{array}$ \\
\hline Brucellosis & 1 & 33.3 & 3 & $\begin{array}{l}\text { Dominican Republic, Iraq, } \\
\text { Peru }\end{array}$ \\
\hline \multicolumn{5}{|l|}{$\begin{array}{l}\text { Chief complaint gastrointestinal } \\
(n=804)\end{array}$} \\
\hline Acute diarrhea $^{\mp}$ & 128 & 96.2 & 133 & $\begin{array}{l}\text { Mexico, India, Dominican } \\
\text { Republic }\end{array}$ \\
\hline $\begin{array}{l}\text { Post-infectious irritable bowel } \\
\text { syndrome }\end{array}$ & 133 & 100.0 & 133 & India, Cuba, Mexico \\
\hline Chronic diarrhea & 122 & 99.2 & 123 & Cuba, India, Mexico \\
\hline Giardiasis & 42 & 87.5 & 48 & India, Cambodia, Ghana \\
\hline Dientamoebiasis & 27 & 90.0 & 30 & Mexico, Dominican Republic \\
\hline Campylobacteriosis & 16 & 100.0 & 16 & $\begin{array}{l}\text { Peru, Indonesia, India, } \\
\text { Cambodia }\end{array}$ \\
\hline Cryptosporidiosis, cyclosporiasis & 8 & 80.0 & 10 & United States, Costa Rica \\
\hline $\begin{array}{l}\text { Amoebiasis due to Entamoeba } \\
\text { histolytica }^{\S}\end{array}$ & 4 & 100.0 & 4 & $\begin{array}{l}\text { Dominican Republic, India, } \\
\text { Turkey }\end{array}$ \\
\hline \multicolumn{5}{|l|}{$\begin{array}{l}\text { Chief complaint dermatologic ( } \mathrm{n} \\
=440 \text { ) }\end{array}$} \\
\hline Skin and soft tissue infection ${ }^{* *}$ & 60 & 72.3 & 83 & Cuba, India, United States \\
\hline Arthropod bite & 77 & 97.5 & 79 & $\begin{array}{l}\text { United States, Dominican } \\
\text { Republic, India }\end{array}$ \\
\hline Insect & 60 & 96.8 & 62 & \\
\hline Tick/Spider & 17 & 100.0 & 17 & \\
\hline Rash & 70 & 95.9 & 73 & $\begin{array}{l}\text { Cuba, Mexico, Dominican } \\
\text { Republic }\end{array}$ \\
\hline Atopic dermatitis & 19 & 100.0 & 19 & \\
\hline Contact dermatitis & 12 & 100.0 & 12 & \\
\hline Drug reaction & 2 & 66.7 & 3 & \\
\hline Photosensitivity reaction & 7 & 100.0 & 7 & \\
\hline Unknown rash & 23 & 100.0 & 23 & \\
\hline Urticarial & 8 & 80.0 & 10 & \\
\hline Cutaneous larva migrans & 43 & 100.0 & 43 & Jamaica, Thailand, Mexico \\
\hline Animal bite $^{\mathrm{T}}$ & 22 & 78.6 & 28 & Thailand, Indonesia, Mexico, \\
\hline
\end{tabular}




\begin{tabular}{l|c|c|c|l} 
& & & & Costa Rica, Chile \\
\hline Cutaneous leishmaniasis & 16 & 100.0 & 16 & $\begin{array}{l}\text { Costa Rica, Mexico, } \\
\text { Afghanistan }\end{array}$ \\
\hline Marine envenomation & 11 & 84.6 & 13 & United States, Mexico \\
\hline
\end{tabular}

*Percentages are calculated by total number in the database. An ill returned traveller could present with more than one chief complaint.

${ }^{\dagger}$ Includes infection with Rickettsia africae, R. conorii, and R. rickettsii.

${ }^{\ddagger}$ Includes acute bacterial, parasitic, and viral diarrhea, as well as acute diarrhea of unspecified cause.

$\S$ Includes both intestinal and extraintestinal amoebiasis.

"Includes lice, fleas, true bugs, mosquitoes, flies, and midges.

"Includes erysipelas, cellulitis, furunculosis, carbuncles, skin abscess, pyoderma, ecthyma, impetigo, and superficial fungal skin infections.

${ }^{\dagger+}$ Includes bites by cats, dogs, monkeys, and other animals.

${ }^{£}$ Where only one or two countries are listed, this indicates $>3$-way tie for second or third place.

Table 4: Cases of malaria among 2004 ill returned travellers with a travel-related diagnosis, by purpose of travel

\begin{tabular}{|c|c|c|c|c|c|c|c|c|c|}
\hline & & & Type of & alaria; no. & cases & & & & \\
\hline $\begin{array}{l}\text { Reason for } \\
\text { travel }\end{array}$ & $\begin{array}{l}\text { Total } \\
\text { cases }\end{array}$ & $\begin{array}{c}P . \\
\text { falciparum }\end{array}$ & $\begin{array}{l}\text { Severe } \\
\text { or } \\
\text { cerebral } \\
\text { malaria }\end{array}$ & P. vivax & P. ovale & $\begin{array}{l}\text { Plasmo- } \\
\text { dium } \\
\text { species } \\
\text { unknown }\end{array}$ & $\begin{array}{l}\text { Top three }^{\mp} \\
\text { countries of } \\
\text { exposure }\end{array}$ & $\begin{array}{l}\text { Obtained } \\
\text { pre-travel } \\
\text { advice }\end{array}$ & $\begin{array}{l}\text { Received } \\
\text { prophy- } \\
\text { laxis }\end{array}$ \\
\hline All $(n=2004)$ & 51 & 30 & 1 & 12 & 2 & 6 & See Table 3 & $\begin{array}{c}10 \\
(19.6)\end{array}$ & $3^{\dagger}$ \\
\hline $\begin{array}{l}\text { Tourism }(n= \\
883)\end{array}$ & 3 & 2 & 0 & 0 & 0 & 1 & $\begin{array}{l}\text { Gabon, } \\
\text { Ghana, } \\
\text { Thailand }\end{array}$ & $\begin{array}{c}2 \\
(66.7)\end{array}$ & 0 \\
\hline $\begin{array}{l}\text { Immigration }(n \\
=493)\end{array}$ & 6 & 2 & 0 & 4 & 0 & 0 & $\begin{array}{c}\text { India, } \\
\text { Afghanistan, } \\
\text { Nigeria, } \\
\text { Gabon }\end{array}$ & $\mathrm{N} / \mathrm{A}$ & $\mathrm{N} / \mathrm{A}$ \\
\hline $\begin{array}{l}\text { Visit friends and } \\
\text { relatives }(n= \\
206)\end{array}$ & 21 & 12 & 0 & 8 & 0 & 1 & $\begin{array}{c}\text { India, } \\
\text { Pakistan, } \\
\text { Nigeria }\end{array}$ & $\begin{array}{c}3 \\
(14.3)\end{array}$ & 1 \\
\hline $\begin{array}{l}\text { Missionary, } \\
\text { volunteer, } \\
\text { researcher, aid } \\
(\mathrm{n}=212) \\
\end{array}$ & 12 & 9 & 1 & 0 & 1 & 1 & $\begin{array}{l}\text { Haiti, Ivory } \\
\text { Coast, } \\
\text { Cameroon }\end{array}$ & $\begin{array}{c}3 \\
(25.0)\end{array}$ & 2 \\
\hline $\begin{array}{l}\text { Business }(n= \\
160)\end{array}$ & 9 & 5 & 0 & 0 & 1 & 3 & Sierra Leone & $\begin{array}{c}2 \\
(22.2)\end{array}$ & 0 \\
\hline $\begin{array}{l}\text { Other* } \\
(n=50)\end{array}$ & 0 & 0 & 0 & 0 & 0 & 0 & $\mathrm{~N} / \mathrm{A}$ & $\mathrm{N} / \mathrm{A}$ & $\mathrm{N} / \mathrm{A}$ \\
\hline
\end{tabular}

NA = not applicable, P. falciparum $=$ Plasmodium falciparum.

*Includes students $(n=42)$, military personnel $(n=6)$ and medical tourists $(n=2)$.

${ }^{\dagger}$ Includes two travellers who either missed doses of doxycycline throughout travel or ran out of doxycycline prior to the end of travel.

‡Where only one or two countries are listed, this indicates >three-way tie for second or third place.

Malaria was the top specific diagnosis for those travelling for the purpose of visiting friends and relatives and was the fifth most common diagnosis among business travellers and missionaries, researchers, volunteers and aid workers (Table 2). Malaria was over-represented among VFR travellers $(p<0.0001)$ and business $(p=0.02)$ compared to other types of travellers. Malaria was also over-represented among males $(p=0.0003)$. Both cases of 
malaria that were diagnosed in ill returned pediatric travellers were caused by $\mathrm{P}$. falciparum and occurred in children travelling for the purpose of visiting friends and relatives (VFR). Of travellers with malaria, $20 \%$ had received pre-travel consultation, yet only three took any course of malaria prophylaxis (Table 4). A full quarter $(\mathrm{N}=13)$ of returned travellers with malaria required inpatient management, compared to only $2.8 \%(\mathrm{~N}=55)$ of those without malaria $(p<0.0001)$. While Sub-Saharan Africa remains the top source region for imported malaria to Canada (33/51 cases; $64.7 \%)$, India was the top specific country of exposure (8/51 cases; $15.7 \%)$. Of business travellers with malaria, 8 of $9(88.9 \%)$ acquired their disease in West Africa or South Sudan.

In addition to malaria, enteric fever was also over-represented among those travelling for the purpose of visiting friends and relatives $(p<0.0001)$ compared to other types of travellers. Cases of enteric fever due to Salmonella enterica serotype Typhi $(\mathrm{N}=2)$ and Paratyphi $(\mathrm{N}=2)$, as well as hepatitis $\mathrm{E}$ virus $(\mathrm{N}=1)$ and hepatitis $A$ virus $(\mathrm{N}=1)$ were all represented among children who were visiting friends and relatives. The proportion of ill returned travellers who had been visiting friends and relatives who required inpatient management of their travel-acquired illness $(12.1 \%)$ was more than five times that of ill returned travellers who had not been visiting friends and relatives $(2.4 \%)(p<0.0001)$. VFR travellers also had the lowest proportionate uptake of pre-travel consultation among all ill returned non-immigrant travellers $(p<0.0001)$ (Table 1). In addition, those who were visiting friends and relatives travelled for longer periods of time compared to travellers who had not visited friends and relatives (median 36 versus 18 days; $p<0.001$ ).

Other emerging, life-threatening and notifiable diseases were represented among the cohort of ill returned travellers. There were nine cases of newly diagnosed or acute (febrile) HIV infection, three of which occurred in non-immigration travellers and a single case of acute hepatitis $B$ in a tourist traveller. Hepatitis $E$ is an emerging infection among travellers and there were six cases diagnosed in this cohort, with $4(67 \%)$ occurring in males, three $(50 \%)$ in VFR travellers and two (33\%) requiring inpatient management. The age range of hepatitis $E$ cases was 4 - 63 years. Five of six cases of hepatitis E virus were acquired in South Central or Southeast Asia. Three cases of brucellosis were diagnosed in immigrant and VFR travellers. Trip duration was known for one case and was 177 days, consistent with the long duration of exposure typically associated with travel-related brucellosis.

Cutaneous larva migrans appears to be an emerging disease among ill returned travellers, with 27 of $43(62.8 \%)$ cases occurring in travellers returning from the Caribbean. Jamaica was the top country of exposure for cutaneous larva migrans, with $18(42 \%)$ cases acquired on this island. Ninety-three percent of cutaneous larva migrans cases ( $\mathrm{N}=40$ ) occurred in tourist travellers on short-stay trips (median trip duration 8.5 days). Of 16 cases of cutaneous leishmaniasis, $69 \%$ were imported from Central or South America and in particular, $50 \%$ were acquired in Costa Rica.

\section{Discussion}

Analysis of surveillance data on ill returned travellers presenting to a CanTravNet site between September 2011 and September 2012 has revealed the spectrum of travel-acquired illness encountered at CanTravNet sites. These data provide an update to the largest surveillance report on illness in Canadian travellers (2009 - 2011) (6).

\section{Potentially life-threatening illnesses can occur in ill returned travellers}

Malaria remains the top specific cause of fever in ill returned travellers $(9,10)$ and in this study, was caused by the potentially life-threatening P. falciparum in $60 \%$ of cases. III returned travellers with malaria were eight-times as likely to require inpatient management compared to those with alternate diagnoses. Malaria is a preventable infection, yet, of patients with malaria who clearly travelled to a risk area, only $20 \%$ sought pre-travel advice and only $6 \%$ received malaria chemoprophylaxis. 
It has been previously demonstrated that travellers are more likely to be exposed to blood and body fluids while travelling than at home $(11,12)$. We noted nine cases of acute or newly diagnosed HIV in our cohort, one third of which occurred in non-immigration travellers, as well as one case of acute hepatitis $B$ in a tourist traveller. Hepatitis $B$, in particular, remains a risk to international travellers, despite being almost completely vaccinepreventable (13-15). Our data reiterate the importance of pre-travel strategies which aim to reduce the likelihood of blood and body fluid exposure while travelling (16), in addition to strategies which mitigate the risk of food-and water-borne and vector-borne diseases.

Animal bites were the tenth most common diagnosis among tourist travellers and were acquired in countries such as Thailand, Indonesia, Mexico, Costa Rica and Chile, which may not have readily available human rabies immune globulin (HRIG) or vaccine that meets minimum potency standards set forth by the World Health Organization (WHO) (17). As rabies is virtually always fatal, ensuring timely access to full post-exposure prophylaxis is essential, yet it is rarely available in rural developing world settings (17). Prevention of animal bites altogether eliminates the risk of rabies and obviates the need for access to post-exposure prophylaxis and, along with rabies pre-immunization, should therefore serve as a target for pre-travel intervention in the consultation setting.

\section{Travellers visiting friends and relatives are at risk}

VFR travellers are known to acquire specific travel-related illnesses more frequently than others, likely because these travellers tend to stay in local homes, travel for longer durations and may fail to recognize the health risks inherent to travel to their country of origin $(3,4,7,8,18)$. Although VFR travellers comprised only $10 \%$ of this cohort, they accounted for $83 \%$ of cases of enteric fever and $41 \%$ of cases of malaria. In addition, the proportion of VFR travellers requiring inpatient management of their travel-acquired illness was five-fold higher than ill returned travellers who had not visited friends and relatives, which may simply reflect that those visiting friends and relatives are more likely to seek care for more serious illness, rather than benign etiologies, as opposed to being more likely to acquire more serious illness. Those who visited friends and relatives travelled for a longer average period of time, yet, they were least likely of all travellers to have sought pre-travel advice. The data underscores the unique characteristics of these of travellers and the urgent need to identify strategies to minimize their travelacquired morbidity.

\section{Emerging infections among travellers are difficult to prevent}

Hepatitis $E$ is a water-borne virus with large epidemics reported from Central America, Sub-Saharan Africa, the Middle East and Asia $(19,20)$. In our cohort, $83 \%$ of cases were acquired from the Indian sub-continent or Southeast Asia and again, those visiting friends and relatives were the most well represented type of traveller, suggesting that, as is the case for hepatitis $A$ and enteric fever (21), more prolonged, rural and in-home travel may be a risk factor for hepatitis $E$ acquisition. Hepatitis $E$ infection is a particular danger for pregnant women (20), especially in the third trimester, with associated maternal mortality as high as $25 \%$ (22). Pregnant travellers should therefore be advised of this risk when travelling to endemic countries (19) and food- and water-precautions should be emphasized.

Chikungunya fever has recently emerged in the Americas (23) and it is anticipated that Canadian physicians will increasingly encounter this disease (24). Prevention of chikungunya rests on mosquito avoidance measures, principally repellents, which are often deemed unappealing by travellers (25-28). Cutaneous larva migrans, while not life-threatening, causes considerable morbidity, lost productivity and costly medical encounters due to the severity of pruritus and lack of ready access to the only effective medications, albendazole and ivermectin, which are not currently licensed in Canada. These drugs can only be acquired in Canada through the Special Access Programme of Health Canada, which necessitates a paper or electronic application for approval and has a 
processing time of at least one week for each request http://www.hc-sc.gc.ca/dhp-mps/acces/drugsdrogues/sapg3 pasg3-eng.php. Prevention of cutaneous larva migrans rests on avoidance of barefoot or bare skin exposure to sand, which is difficult to achieve in beach destinations where the causative organism is prevalent. Cutaneous leishmaniasis, an emerging vector-borne disease among travellers (29), is challenging to prevent as it requires absolute bite avoidance in destinations with typically high ambient temperatures and humidity, where the use of long clothing and repellents might be deemed inconvenient (25-28). Insecticide treated bed nets and sleeping several feet above the ground may provide some protection.

\section{Limitations}

Analysis of CanTravNet data has several limitations. This report focuses only on those ill returned travellers who presented to a CanTravNet centre, thus, conclusions may lack external validity. It must be noted that many of the top illnesses in those travelling for the purpose of immigration, including latent tuberculosis, hepatitis $B$ and hepatitis $\mathrm{C}$, would have been diagnosed through screening of at-risk individuals from endemic areas and cannot be definitively linked to travel. Travellers with illnesses with very short or very long incubation periods may have sought care in different settings and these diagnoses are difficult to definitively link to travel. Similarly, ill travellers returning from destinations perceived to be low-risk may be under-represented in the CanTravNet database. The data cannot estimate incidence rates or destination-specific numerical risks for particular diseases $(7,30)$. Variation among sites regarding screening protocols for new immigrants and refugees may have led to over- or under-contributions of particular diagnoses from individual sites. Fifty-three percent of cases were contributed by Montreal sites, which may have introduced bias given the inter-Provincial variation in travel patterns and preferences.

\section{Conclusions}

This surveillance report aims to better inform pre- and post-travel management and to illuminate changing patterns of imported diseases. Malaria remains the top specific diagnosis among travellers visiting friends and relatives and although still mostly acquired in Sub-Saharan Africa, India was the top single source country of imported malaria in this cohort. In addition to malaria, other preventable travel-acquired illnesses such as enteric fever, influenza, hepatitis B and animal bites were common and reinforce that improved translation of knowledge into action on the part of the traveller should be prioritized. In addition, barriers to the uptake of pre-travel consultation by particular risk groups, such as VFR travellers and barriers to the use of preventive interventions, such as insect repellent and malaria chemoprophylaxis, should be assessed in the travelling Canadian population.

\section{Conflict of interest}

There are no conflicts of interest to declare.

\section{References}

(1) World Tourism Organization. UNWTO tourism highlights, 2013 Edition. http://www.unwto.org

(2) Statistics Canada. Travel by Canadians to foreign countries: Top 15 countries visited. Statistics Canada. Tourism and the Centre for Education Statistics, 2012. http://www.statcan.gc.ca/tablestableaux/sum-som/l01/cst01/arts37a-eng.htm

(3) Ryan ET, Wilson ME, Kain KC. Illness after international travel. N Eng J Med 2002;347:505-516. 
(4) Ryan ET, Kain KC. Health advice and immunizations for travelers. N Eng J Med 2000;342:17161725.

(5) Steffen R, Rickenbach M, Wilhelm U, Helminger A, Schar M. Health problems after travel to developing countries. J Infect Dis 1987;156:84-91.

(6) Boggild AK, Geduld J, Libman M, McCarthy A, Doyle P, Ghesquiere W, Vincelette J, Kuhn S, Freedman DO, Kain KC. Travel-acquired infections and illnesses in Canadians: surveillance report from CanTravNet surveillance data, 2009-2011. Open Medicine 2014; 8(1):e20-e32.

(7) Freedman DO, Weld LH, Kozarsky PE, Fisk T, Robins R, von Sonnenburg F, et al. Spectrum of disease and relation to place of exposure among ill returned travelers. N Eng J Med 2006;354:119-130.

(8) Leder K, Tong S, Weld L, Kain KC, Wilder-Smith A, von Sonnenburg F, et al. Illness in travelers visiting friends and relatives: a review of the GeoSentinel Surveillance Network. Clin Infect Dis 2006;43:1185-1193.

(9) Boggild AK, Ghesquiere W, McCarthy A, for the Committee to Advise on Tropical Medicine and Travel (CATMAT). Fever in the Returning International Traveller: Initial Assessment Guidelines. Can Commun Dis Rep 2011; 37(ACS-3):1-15.

(10) Committee to Advise on Tropical Medicine and Travel (CATMAT). Canadian recommendations for the prevention and treatment of malaria among international travellers. Can Commun Dis Rep 2009;35(S1):1-82. http://www.phac-aspc.gc.ca/publicat/ccdr-rmtc/09vol35/35s1/index-eng.php

(11) Salit IE, Sano M, Boggild AK, Kain KC. Travel patterns and risk behaviour of HIV-positive people travelling internationally. CMAJ 2005;172:884-888.

(12) Correia JD, Shafer RT, Patel V, Kain KC, Tessier D, MacPherson D, et al. Blood and body fluid exposures as a health risk for international travellers. J Travel Med 2001;8:263-266.

(13) Zuckerman JN, Stephen R. Risk of hepatitis B in travelers as compared to immunization status. J Travel Med 2000;7:170-174.

(14) Connor BA, Jacobs RJ, Meyerhoff AS. Hepatitis B risks and immunization coverage among American travellers. J Travel Med 2006;13:273-280.

(15) Streeton CL, Zwar N. Risk of exposure to hepatitis B and other blood-borne viruses among Australians who travel abroad. J Travel Med 2006;13:345-350.

(16) Ward BJ, Plourde P, for the Committee to Advise on Tropical Medicine and Travel (CATMAT). Statement on travellers and sexually transmitted infections. Can Commun Dis Rep 2006; Volume 32(ACS-5):1-24.

(17) Jentes ES, Blanton JD, Johnson KJ, et al. The global availability of rabies immune globulin and rabies vaccine in clinics providing direct care to travelers. J Travel Med 2013;20(3):148-158.

(18) Wilson ME, Weld LH, Boggild A, Keystone JS, Kain KC, von Sonnenburg F, et al. Fever in returned travellers: Results from the GeoSentinel Surveillance Network. Clin Infect Dis 2007;44:1560-1568. 
(19) Teo C-G. Hepatitis E. Chapter 3, Infectious Diseases Related to Travel. IN: CDC Health Information for International Travel. Centers for Disease Control and Prevention, Atlanta. http://wwwnc.cdc.gov/travel/yellowbook/2014/chapter-3-infectious-diseases-related-totravel/hepatitis-e (accessed February 28, 2014)

(20) Kamar N, Dalton HR, Abravanel F, Izopet J. Hepatitis E Virus Infection. Clin Microbiol Rev 2014;27(1):116.

(21) Bui YG, Trépanier S, Milord F, Blackburn M, Provost S, Gagnon S. Cases of malaria, hepatitis A and typhoid fever among VFRs, Quebec (Canada). J Travel Med. 2011;18:373-378.

(22) Labrique A, Kuniholm MH, Nelson K. 2010. The global impact of hepatitis E: new horizons for an emerging virus, p 53-93. In Scheld WM, Grayson ML, Hughes JM (ed), Emerging infections, 9th ed. ASM Press, Washington, DC.

(23) Chikungunya outbreak in the Caribbean, 2013-2014. Public Health Agency of Canada. www.phacaspc.gc.ca/publicat/ccdr-rmtc /14 vol40/dr-rm40 -02 /dr-rm40-02-chik-eng .php

(24) Schwartz K, Giga A, Boggild AK. Chikungunya Fever in Canada: Fever and Polyarthritis in a Returned Traveler. CMAJ 2014; e-pub before print 24-Feb-2014 DOI:10.1503/cmaj.130680

(25) Schofield S, Plourde P, for the Committee to Advise on Tropical Medicine and Travel (CATMAT). Statement on Personal Protective Measures to Prevent Arthropod Bites. Can Commun Dis Rep 2012; 38(ACS-3):1-18.

(26) Vickery JP, Tribble DR, Putnam SD, McGraw T, Sanders JW, Armstrong AW, et al. Factors associated with the use of protective measures against vector-borne diseases among troops deployed to Iraq and Afghanistan. Mil Med 2008;173(11):1060-7.

(27) Keystone JS. Compliance with travel health recommendations. In: Dupont HI, Steffen F, editors. Textbook of travel medicine and health. London: B.C. Decker Inc.; 2001.

(28) Alon D, Shitrit P, Chowers M. Risk behaviors and spectrum of diseases among elderly travelers: A comparison of younger and older adults. J Travel Med 2010;17(4):250-5.

(29) Pavli A, Maltezou HC. Leishmaniasis, an emerging infection in travelers. Int J Infect Dis 2010;14(12):e1032-9.

(30) Leder K, Wilson ME, Freedman DO, Torresi J. A comparative analysis of methodological approaches used for estimating risk in travel medicine. J Travel Med 2008;15:263-72. 